\title{
Antioxidant capacity and polyphenolic composition as quality indicators for aqueous infusions of Salvia officinalis L. (sage tea)
}

\author{
Stephan G. Walch ${ }^{1,2}$, Laura Ngaba Tinzoh ${ }^{3}$, Benno F. Zimmermann ${ }^{3,4}$, Wolf Stühlinger $^{1}$ and \\ Dirk W. Lachenmeier ${ }^{5}$
}

${ }^{1}$ Department for Public Health and Health Technology Assessment, University for Health Sciences, Medical Informatics and Technology, Hall in Tyrol, Austria

2 Zentrales Institut des Sanitätsdienstes der Bundeswehr München, Garching-Hochbrück, Germany

3 Institut Prof. Dr. Kurz GmbH, Köln, Germany

${ }^{4}$ Department of Food Chemistry, Institute of Nutrition and Food Sciences, University of Bonn, Bonn, Germany

${ }^{5}$ Chemisches und Veterinäruntersuchungsamt Karlsruhe, Karlsruhe, Germany

\section{Edited by:}

Dong Xiao, University of Pittsburgh, USA

\section{Reviewed by:}

Haizhong Feng, University of Pittsburgh, USA

Caisheng Lu, University of Pittsburgh Cancer Institute, USA

Ajay Bommareddy, Wilkes University, USA

*Correspondence:

Dirk W. Lachenmeier, Chemisches und Veterinäruntersuchungsamt Karlsruhe, Weissenburger Strasse 3, D-76187 Karlsruhe, Germany e-mail: lachenmeier@web.de

\begin{abstract}
Sage (Salvia officinalis L.) is used as an herbal medicinal product, with the most typical form of application as infusion with boiling water (sage tea). The well-established traditional uses include symptomatic treatment of mild dyspeptic complaints, the treatment of inflammations in the mouth and the throat, and relief of excessive sweating and relief of minor skin inflammations. In this study, sage teas prepared from commercially available products were chemically analyzed for polyphenolic content using liquid chromatography, for antioxidant potential using the oxygen radical absorbance capacity method, and for the Folin-Ciocalteu (FC) index. The sage teas showed a high variation for all parameters studied (up to 20-fold differences for rosmarinic acid). Univariate and multivariate analyses showed that the antioxidant potential, which varied between 0.4 and $1.8 \mathrm{mmol}$ trolox equivalents $/ 100 \mathrm{~mL}$, was highly dependent on rosmarinic acid and its derivatives. The FC index also showed a high correlation to these polyphenols, and could therefore be used as a screening parameter for sage tea quality. The considerable differences in polyphenolic composition and antioxidant capacity between the brands lead to a demand for quality standardization, especially if these sage teas are to be used for therapeutic purposes. Further research also appears to be necessary to characterize the dose-benefit relationship, as sage may also contain a constituent (thujone) with potentially adverse effects.
\end{abstract}

Keywords: Salvia officinalis L., tea infusion, polyphenols, antioxidant capacity, ORAC

\section{INTRODUCTION}

Sage (Salvia officinalis L.) has been an important herbal medicinal product since very early times and is still in wide use today. An infusion of dried sage leaves with boiling water (sage tea) is the most typical form of preparation (Dweck, 2000). Although wellestablished traditional uses of sage include symptomatic treatment of mild dyspeptic complaints, the treatment of inflammations in the mouth and the throat, and relief of excessive sweating and minor skin inflammations, only a few clinical trials thus far have been conducted to corroborate these uses.

The European Medicines Agency (EMA) reported a number of unpublished studies confirming the perspiration-inhibiting and antihidrotic effects of sage (European Medicines Agency, 2009). The efficacy of sage for the treatment of hot flushes during menopause was proven by a multicentre open clinical trial (Bommer et al., 2009). A statistically significant effect of symptomatic relief in patients with acute pharyngitis was detected (Hubbert et al., 2006). In addition, sage combined with Echinacea was efficacious in the treatment of acute sore throats (Schapowal et al., 2009). A double-blind, randomized, and placebo-controlled trial also indicated that sage may improve the symptoms of Alzheimer's disease (Akhondzadeh et al., 2003). Sage tea was effective in the improvement of lipid profile, antioxidant defenses, and lymphocyte Hsp70 protein expression in human volunteers (Sá et al., 2009).

Animal experiments and in vitro studies have substantiated that Salvia officinalis extracts may significantly decrease serum glucose in diabetic rats (Eidi et al., 2005) and positively affect the antioxidant status of the liver (Lima et al., 2005). Sage may also inhibit pro-oxidant-induced lipid peroxidation in rat brain and liver homogenates (Oboh and Henle, 2009). Further in vitro models showed Salvia as possibly being antiangiogenic, antimutagenic, antidiabetic, and gastroprotective (Lima et al., 2006; Mayer et al., 2009; Patenkovic et al., 2009; Keshavarz et al., 2010). Although the underlying mechanisms of action are unknown, polyphenols such as carnosol, carnosic acid, rosmanol, apigenin, hispidulin, caffeic acid, and ursolic acid have been discussed as active compounds for these pharmacological effects (Imanshahidi and Hosseinzadeh, 2006). An excellent review on sage polyphenols was provided by Lu and Foo (2002).

We have previously discussed the potentially adverse effects of sage attributed to the presence of the neurotoxic monoterpene 
compound thujone; however, this compound was found to be below thresholds of toxicity at normal levels of use (Lachenmeier and Uebelacker, 2010; Walch et al., 2011). In this contribution, we have focused on the beneficial effects of sage due to antioxidant capacity and polyphenol content, as analyzed by a recently developed methodology (Zimmermann et al., 2011). It is interesting that neither the European Pharmacopoeia (2008) monograph nor the ISO (1995) standard 11165 on sage demand a standardization regarding the content of polyphenols and antioxidant capacity, and a general lack of knowledge on these constituents exists. Earlier studies provided information of only single samples of herbal sage tea (Aoshima et al., 2007; Hossain et al., 2011). By analyzing different sage tea brands from the German market, we will try to determine whether considerable differences in tea brands exist and if these differences are related to product quality. We will also investigate the contribution of polyphenolic constituents to the antioxidant capacity measured by the oxygen radical absorbance capacity (ORAC) method.

\section{MATERIALS AND METHODS SAMPLE PREPARATION}

Infusions were prepared in a 250-mL Erlenmeyer flask by adding $150 \mathrm{~mL}$ boiling water to one tea bag (containing $1.5 \mathrm{~g}$ ) or $1.5 \mathrm{~g}$ of herbal sage material in the case of loose tea. The infusion was allowed to steep for $15 \mathrm{~min}$ and was shaken every $5 \mathrm{~min}$. All samples were prepared as delivered, e.g., whole leafs were not crushed. One milliliter of the infusion was filtered through a disposable polyester filter (Chromafil PET-20/25, Macherey-Nagel, Düren, Germany) with $0.20 \mu \mathrm{m}$ pore size and injected into the HPLC system within 20 min or diluted immediately for the ORAC and Folin-Ciocalteu (FC) analyses.

\section{APPARATUS AND SOFTWARE FOR LC-MS/MS MEASUREMENT}

Method development, compound identification, quantification, and validation was previously described in detail (Zimmermann et al., 2011). In short, an Acquity UPLC system by Waters (Milford, MA, USA) consisting of a binary pump (BSM), an autosampler (SM; cooled to $10^{\circ} \mathrm{C}$; injected volume: $3 \mu \mathrm{L}$ ), a column oven $(\mathrm{CM})$ set at $40^{\circ} \mathrm{C}$, a diode array detector (PDA) scanning from 190 to $400 \mathrm{~nm}$ (wavelengths for quantification: 273, 320, and $360 \mathrm{~nm}$ ), and a triple quadrupole mass spectrometer (Acquity TQD) with electrospray interface operating in negative mode was used. An Acquity BEH Shield RP18 column $(150 \mathrm{~mm} \times 2.1 \mathrm{~mm}, 1.7 \mu \mathrm{m}$; Waters) was used for separation with water (A) and acetonitrile (B) as eluents, both acidified with $0.1 \%(\mathrm{v} / \mathrm{v})$ formic acid at a flow rate of $0.4 \mathrm{~mL} / \mathrm{min}$ with gradient elution: $0 \mathrm{~min}: 4 \% \mathrm{~A}, 14 \mathrm{~min}$ : $27 \%$ A, $28 \mathrm{~min}: 59.7 \%$ A, $28.2 \mathrm{~min}: 100 \%$ A, $30.5 \mathrm{~min}: 100 \% \mathrm{~A}$, $31 \mathrm{~min}$ : 4\% A, $34 \mathrm{~min}: 4 \%$ A.

The mass spectrometer was tuned using a solution of pure rosmarinic acid and luteolin-7-O-glucuronide. The resulting parameters for the measurements were a compromise between the optimal parameters for these compounds: capillary voltage $-2.0 \mathrm{kV}$, cone voltage $30 \mathrm{~V}$, extractor voltage $1.0 \mathrm{~V}$, RF voltage $0.20 \mathrm{~V}$, source temperature $150^{\circ} \mathrm{C}$, desolvation temperature $400^{\circ} \mathrm{C}$, cone gas (nitrogen) flow $50 \mathrm{~L} / \mathrm{h}$, desolvation gas (nitrogen) flow $800 \mathrm{~L} / \mathrm{h}$. The collision gas (argon) flow in tandem mass spectrometry mode was $0.3 \mathrm{~mL} / \mathrm{min}$. The whole system was controlled by MassLynx
4.1 software. Averages from analysis in duplicate are reported. The relative SD was $5.0 \%$ on average. For full validation results see Zimmermann et al. (2011).

\section{DETERMINATION OF ANTIOXIDANT CAPACITY AND FC INDEX}

The ORAC method was used to determine the antioxidant capacity of the tea samples (Prior et al., 2003). Reagents: 2,2'-azobis(2-amidino-propane)-dihydrochloride (AAPH; $240 \mathrm{mM})$, 6hydroxy-2,5,7,8-tetra-methylchroman-2-carboxylic acid (trolox; $100-6.25 \mu \mathrm{M})$, fluorescein sodium salt $(10 \mathrm{nM})$. All chemicals were obtained by Aldrich/Sigma-Aldrich (Taufkirchen, Germany) and dissolved in phosphate buffer $\mathrm{pH} 7.4,10 \mathrm{mM}$.

The fluorescence was measured by a 96 -well plate reader (FLUOstar Optima, BMG Labtech, Offenburg, Germany). Every solution was pipetted in duplicate: $25 \mu \mathrm{L}$ of trolox dilution as reference, $25 \mu \mathrm{L}$ of sample dilution, and $25 \mu \mathrm{L}$ of phosphate buffer as blank. After an incubation period of $30 \mathrm{~min}$ at $37^{\circ} \mathrm{C}$, three fluorescence measurements (excitation $485 \mathrm{~nm}$, emission $520 \mathrm{~nm}$ ) were taken every $90 \mathrm{~s}$ to determine the background. After that, AAPH was added, and fluorescence was measured every $90 \mathrm{~s}$ for $60 \mathrm{~min}$. Data were evaluated using the MARS software, version 2.0 (BMG Labtech). Averages from analysis in duplicate are reported. The relative SD was $4.9 \%$ on average.

The FC test was used to further characterize the samples. The FC index was determined according to the reference procedure for wine analysis (European Commission, 1990) using a commercial FC reagent (Merck, Darmstadt, Germany, No. 1.09001.0100). $200 \mu \mathrm{L}$ of sage tea (from the infusion described above), $10 \mathrm{~mL}$ of distilled water, $1 \mathrm{~mL}$ of FC reagent, and $4 \mathrm{~mL}$ of sodium carbonate solution $(2 \mathrm{~g} / \mathrm{L})$ were placed into a calibrated 20 - $\mathrm{mL}$ flask and filled up to the mark with distilled water. The absorbance was measured at $750 \mathrm{~nm}$ after $30 \mathrm{~min}$. The result of the test was expressed in the form of an index obtained by multiplying the absorbance by 20 . Averages from analysis in triplicate are reported. The relative SD was $3.5 \%$ on average.

\section{DATA ANALYSIS}

All data were evaluated using Origin V7.5 (Originlab, Northampton, MA, USA). Statistical significance was assumed at below the 0.05 probability level. Pearson's test was used to evaluate the significance of linearity of calibration curves as well as of the correlation between different parameters. For explorative multivariate data analysis aiming to visualize the data set, the software Unscrambler $\mathrm{X}$ version 10.0.1 (Camo Software AS, Oslo, Norway) was used. The multivariate evaluation was conducted using principal component analysis (PCA) based on a data matrix containing all substances from LCMS as well as ORAC and FC index values. Standardization was used prior to PCA to give all variables the same variance.

\section{RESULTS}

At first sight, the commercial teas appeared to differ notably in their polyphenolic profiles as well as ORAC and FC values (Table 1). In the polyphenols analysis, the dominating compounds were either rosmarinic acid or luteolin-7-O-glucuronide. The concentrations ranged from 12.2 to $296 \mathrm{mg} / \mathrm{L}$ (rosmarinic acid) and from 37.9 to 


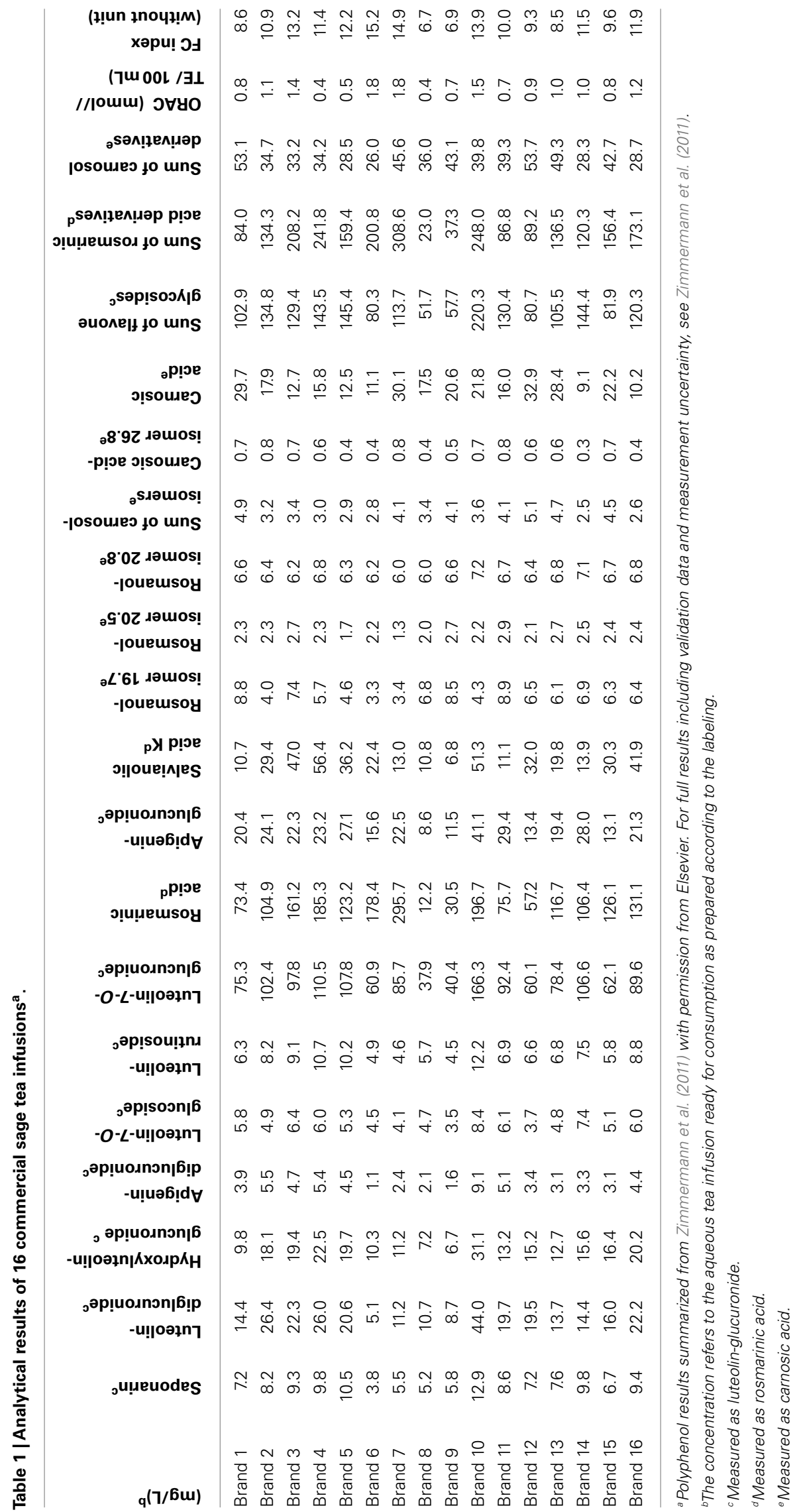




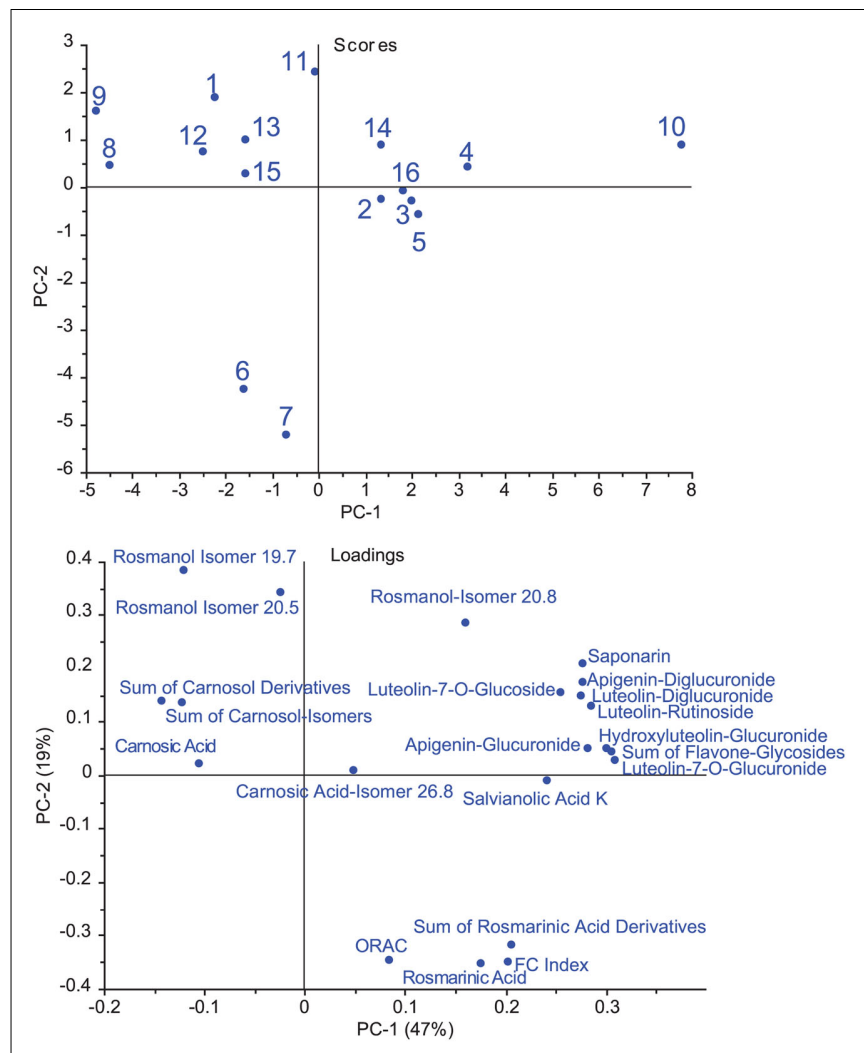

FIGURE 1 | Explorative data analysis of the analytical results of 16 commercial sage tea infusions. The principal component analysis (PCA) plots show the scores (sample numbers 1-16; upper panel) and loadings (names of analytes; lower panel).

$166 \mathrm{mg} / \mathrm{L}$ (luteolin-7-O-glucuronide). Amongst the triterpenes, carnosic acid had the highest concentrations, ranging from 9.1 to $32.9 \mathrm{mg} / \mathrm{L}$. In all but two samples the sum of the concentrations of the caffeic acid derivatives was higher than the sum of the concentrations of the triterpenes. The ORAC values ranged between 0.4 and $1.8 \mathrm{mmol}$ Trolox Equivalents (TE)/100 mL, whereas the FC index varied between 6.7 and 15.2. In all cases, the differences between the tea brands cannot be explained by the uncertainty of the analytical methods.

The results of the explorative data analysis is shown in Figure 1. The PCA scores confirm that a considerable difference exists in the composition of the commercial sage tea samples. Samples 6 and 7 were different from the rest of the samples, especially regarding to their high antioxidative potential. Furthermore, both samples had a high content of polyphenols and high levels of rosmarinic acid and its derivatives. Sample 10 was peculiar not only for higher rosmarinic acid and ORAC levels but especially for its high levels of flavone glycosides, which made it different from all other samples. The PCA loadings plot suggests a correlation between ORAC, FC index, and rosmarinic acid and its derivatives. This finding is confirmed by univariate linear correlation analysis (FC index and rosmarinic acid, $R=0.86 p<0.0001$; ORAC and rosmarinic acid, $R=0.68$ $p=0.00355$ ).

\section{DISCUSSION}

Our analytical results showed a higher variability of the composition of sage teas than what we actually had expected. For example, the content of rosmarinic acid varied by more than a factor of 20 between the lowest and highest brands of sage tea. This shows that it is important to analyze a comprehensive number of samples rather than single samples as it has been done in previous studies (Aoshima et al., 2007; Hossain et al., 2011). The difference in polyphenols content is also evidenced by the corresponding antioxidative potential, which varied from 0.4 to $1.8 \mathrm{mmol} \mathrm{TE} / 100 \mathrm{~mL}$. The information on the packages did not provide details about the plant origins or the exact composition, making it difficult for us to explain the (possible) factors leading to these differences. The brands with the highest ORAC and FC index (samples 6 and 7) were labeled to be hand-collected shoot apex material from organic farming (sample 6) or sage tea selected from shoot apex material (sample 7). This could explain the difference between these two samples and the other tea samples, which appear mainly to be composed of leaf material. Previous research on FC index, thujone and camphor (Walch et al., 2011) has reported results from several batches of a single manufacturer, which were very consistent. For example, the FC index for five different batches of shoot apex material (corresponding to sample 6 in this study) was $14.9 \pm 0.3$. Similar to the results in this study for polyphenols, ORAC, and FC index, the variations for thujone and camphor were significantly higher between manufacturers than within a single brand. This indirect evidence points to sage variety and chemotype, cultivation conditions, climate, or processing conditions (especially during drying) as explanations for the differences rather than pure inter-batch variations.

It is interesting to note that sage tea not only is available as an herbal medicinal product but is also sold as a food product in many supermarkets, as it may simply be consumed as a "pleasant drink" for culinary purposes (Dweck, 2000; British Herbal Compendium, 2006). There was no distinct difference between the teas marketed as medicine (samples 1-13) and those marketed as food (samples 14-16). In contrast, the brands with the lowest ORAC (samples 4, 5, and 8) were all marketed as medicine, whereas the food teas showed an average composition.

Our results confirm that it may be worthwhile to explore sage as a food ingredient not only for its flavor profile but also for its antioxidant capacity so that it may be a substitute for the synthetic antioxidants that are commonly applied in foods (Madsen and Bertelsen, 1995).

According to Wang et al. (1998), the compounds in sage with the highest antioxidant capacity were found to be rosmarinic acid and luteolin-7-O- $\beta$-glucopyranoside, of which rosmarinic acid quantitatively dominated in our samples.

As mentioned in the introduction, there are no legal specifications regarding these compounds in sage tea, which may be an explanation for the obvious lack of quality awareness in this regard. From a consumer's perspective, it is surely a mandatory demand that medicines contain the active principles in a defined amount. In the case of sage (as for many other traditional herbal medicines), the active principle is not known. However, at least the main components that have a hypothesized effect, such as polyphenols or antioxidants, should be regulated. In their Salvia officinalis 
monograph, the EMA - the regulatory authority in Europe - justified an acceptable daily intake (ADI) of thujone at $5.0 \mathrm{mg} /$ person for a maximum duration of use of 2 weeks (European Medicines Agency, 2009). Lately, the EMA has reconsidered this ADI in a public statement (European Medicines Agency, 2011). Nonetheless, if the regulatory authority holds the opinion that a certain risk seems to be involved in the usage of sage as medicine, it is difficult to understand why minimum requirements of the possibly beneficiary compounds, have not yet been established. In times when the public is discussing the financing of healthcare and the quality and the benefits of medicines have to be evaluated, it seems important for the credibility of herbal medicines to establish specifications. Therefore, a dose-benefit assessment appears to be necessary, which could be followed by minimum requirements of the active principles. The FC index appears to be useful as a screening parameter for sage tea quality, as it is highly correlated with rosmarinic acid and easier to determine with less instrumental effort than the ORAC value or the specific compounds using HPLC.

\section{CONCLUSION}

Currently, no standardization or quality control of polyphenolic compounds or antioxidant potential, which may be associated

\section{REFERENCES}

Akhondzadeh, S., Noroozian, M., Mohammadi, M., Ohadinia, S., Jamshidi, A. H., and Khani, M. (2003). Salvia officinalis extract in the treatment of patients with mild to moderate Alzheimer's disease: a double blind, randomized and placebo-controlled trial. J. Clin. Pharm. Ther. 28, 53-59.

Aoshima, H., Hirata, S., and Ayabe, S. (2007). Antioxidative and antihydrogen peroxide activities of various herbal teas. Food Chem. 103, 617-622.

Bommer, S., Klein, P., and Suter, A. (2009). A multicentre open clinical trial to assess the tolerability and efficacy of sage tablets in menopausal patients with hot flushes. Planta Med. 75, 1070.

British Herbal Compendium. (2006). British Herbal Compendium, Vol. 2. Surrey: B. H. M. A. Publishing.

Dweck, A. C. (2000). "The folklore and cosmetic use of various Salvia species," in SAGE - The Genus Salvia, ed. S. E. Kintzios (Amsterdam: Overseas Publishers Association), 1-25.

Eidi, M., Eidi, A., and Zamanizadeh, H. (2005). Effect of Salvia officinalis L. leaves on serum glucose and insulin in healthy and streptozotocin-induced diabetic rats. J. Ethnopharmacol. 100, 310-313.

European Commission. (1990). Commission Regulation (EC) No 2676/90 determining community methods for the analysis of

with the beneficial effects of sage, are conducted. In addition the commercially available products are of highly variable composition. The plant material used in previous in vitro or in vivo studies was in most cases not characterized in this regard (e.g., regarding content of rosmarinic acid or antioxidant potential): therefore the transferability of study results to commercial material per se remains questionable. Nevertheless, the limited available in vivo evidence suggests that sage tea drinking may improve lipid profile and antioxidant defenses in humans (Sá et al., 2009). Further research appears to be necessary to better characterize the dose-benefit relationship and to postulate minimum quality standards for medicinal sage tea that would guarantee the ingestion of effective dosages. The commercial quality could be improved by introducing minimum requirements into the European Pharmacopoeia (2008) monograph or the ISO 11165 standard.

\section{ACKNOWLEDGMENTS}

Hannelore Heger, Christina Diehl, and Jeanne Mildenberger are thanked for their excellent technical assistance including sample preparation and analysis of FC index. No funding was specific to the production of this manuscript. The salaries for authors were provided by the affiliated organizations.

ISO. (1995). ISO 11165. Dried sage (Salvia officinalis L.) - specification. Geneva: International Organization for Standardization.

Keshavarz, M., Mostafaie, A., Mansouri, K., Bidmeshkipour, A., Motlagh, H. R. M., and Parvaneh, S. (2010). In vitro and ex vivo antiangiogenic activity of Salvia officinalis. Phytother. Res. 24, 1526-1531.

Lachenmeier, D. W., and Uebelacker, M. (2010). Risk assessment of thujone in foods and medicines containing sage and wormwood - evidence for a need of regulatory changes? Regul. Toxicol. Pharmacol. 58, 437-443.

Lima, C. F., Andrade, P. B., Seabra, R. M., Fernandes-Ferreira, M., and PereiraWilson, C. (2005). The drinking of a Salvia officinalis infusion improves liver antioxidant status in mice and rats. J. Ethnopharmacol. 97, 383-389.

Lima, C. F., Azevedo, M. F., Araujo, R., Fernandes-Ferreira, M., and PereiraWilson, C. (2006). Metformin-like effect of Salvia officinalis (common sage): is it useful in diabetes prevention? Br. J. Nutr. 96, 326-333.

Lu, Y. R., and Foo, L. Y. (2002). Polyphenolics of Salvia - a review. Phytochemistry 59, 117-140.

Madsen, H. L., and Bertelsen, G. (1995) Spices as antioxidants. Trends Food Sci. Technol. 6, 271-277.

Mayer, B., Baggio, C. H., Freitas, C. S., dos Santos, A. C., Twardowschy, A., Horst, H., Pizzolatti, M. G.,
Micke, G. A., Heller, M., dos Santos, E. P., Otuki, M. F., and Marques, M. C. (2009). Gastroprotective constituents of Salvia officinalis L. Fitoterapia 80, 421-426.

Oboh, G., and Henle, T. (2009). Antioxidant and inhibitory effects of aqueous extracts of Salvia officinalis leaves on pro-oxidant-induced lipid peroxidation in Brain and liver in vitro. J. Med. Food 12, 77-84.

Patenkovic, A., Stamenkovic-Radak, M., Banjanac, T., and Andjelkovic, M. (2009). Antimutagenic effect of sage tea in the wing spot test of Drosophila melanogaster. Food Chem. Toxicol. 47, 180-183.

Prior, R. L., Hoang, H., Gu, L. W., Wu, X. L., Bacchiocca, M., Howard, L., Hampsch-Woodill, M., Huang, D. J., Ou, B. X., and Jacob, R. (2003). Assays for hydrophilic and lipophilic antioxidant capacity (oxygen radical absorbance capacity (ORAC(FL)) of plasma and other biological and food samples. J. Agric. Food Chem. 51, 3273-3279.

Sá, C. M., Ramos, A. A., Azevedo, M. F., Lima, C. F., Fernandes-Ferreira, M., and Pereira-Wilson, C. (2009). Sage tea drinking improves lipid profile and antioxidant defences in humans. Int. J. Mol. Sci. 10, 3937-3950.

Schapowal, A., Berger, D., Klein, P., and Suter, A. (2009). Echinacea/sage or chlorhexidine/lidocaine for treating acute sore throats: a randomized double-blind trial. Eur. J. Med. Res. 14, 406-412. 
Walch, S. G., Kuballa, T., Stuhlinger, W., and Lachenmeier, D. W. (2011). Determination of the biologically active flavour substances thujone and camphor in foods and medicines containing sage (Salvia officinalis L.). Chem. Cent. J. 5, 44.

Wang, M. F., Li, J. G., Rangarajan, M., Shao, Y., Lavoie, E. J., Huang, T. C., and Ho, C. T. (1998). Antioxidative phenolic compounds from sage (Salvia officinalis). J. Agric. Food Chem. 46, 4869-4873.
Zimmermann, B. F., Walch, S. G., Ngaba Tinzoh, L., Stühlinger, W., and Lachenmeier, D. W. (2011). Rapid UHPLC determination of polyphenols in aqueous infusions of Salvia officinalis L. (sage tea). J. Chromatogr. B Analyt. Technol. Biomed. Life Sci. 879, 2459-2464.

Conflict of Interest Statement: The authors declare that the research was conducted in the absence of any commercial or financial relationships that could be construed as a potential conflict of interest.

Received: 02 November 2011; paper pending published: 11 November 2011; accepted: 22 November 2011; published online: 19 December 2011.

Citation: Walch SG, Tinzoh LN, Zimmermann BF, Stühlinger W and Lachenmeier DW (2011) Antioxidant capacity and polyphenolic composition as quality indicators for aqueous infusions of Salvia officinalis L. (sage tea). Front. Pharmacol. 2:79. doi: 10.3389/fphar.2011.00079

This article was submitted to Frontiers in Ethnopharmacology, a specialty of Frontiers in Pharmacology.

Copyright (c) 2011 Walch, Tinzoh, Zimmermann, Stühlinger and Lachenmeier. This is an open-access article distributed under the terms of the Creative Commons Attribution Non Commercial License, which permits non-commercial use, distribution, and reproduction in other forums, provided the original authors and source are credited. 